\title{
INTERACTIONS OF PLANT BIOACTIVES WITH NUTRIENT TRANSPORT SYSTEMS IN GUT OF LIVESTOCK
}

\author{
A. K. PATRA \\ Department of Animal Nutrition \\ West Bengal University of Animal and Fishery Sciences \\ 37 K. B. Sarani, Belgachia, Kolkata- 700 037, India
}

\begin{abstract}
Research on natural plant secondary compounds (PSC) has gained widespread impetus for use as feed additives to improve production performance, welfare and health of livestock and poultry due to their antimicrobial, antioxidant, immunostimulatory and other beneficial biological effects. Moreover, various PSC are usually present in regular diets of animals and humans. Dietary PSC may regulate absorption of nutrients, minerals and ammonia via transcellular and paracellular routes in the gastrointestinal (GI) tract influencing nutrient transporter gene expression and molecular structures of tight junctions. Some PSC stimulate nutrient absorption, while other PSC may impair transport mechanisms in the intestine interacting with nutrient transporters of enterocytes and tight junction motifs and their regulatory proteins. One challenging aspect is to select an effective dose at which a specific PSC could improve GI nutrient absorption while preserving or improving other beneficial biological effects. The optimum doses and precise molecular mechanisms for PSC are yet to be identified to understand discrepant observations among different studies and to improve the targeted biotechnological and pharmaceutical uses of PSC in farm animals. This review discusses the effects of different PSC on nutrient transport and permeability of GI epithelia and their mechanism of actions focusing mainly on livestock species.
\end{abstract}

Key words: Gastrointestinal tract, Ion channel, Nutrient absorption, Permeability, Plant secondary compound

Herbs or their plant secondary compounds (PSC) have gained extensive research interests in recent years for use in livestock and poultry as an alternative to antibiotic feed additives (Windisch et al., 2008; Patra and Saxena, 2009a,b; Pathak et al., 2016; Chowdhury et al., 2018). Many PSC are also normally present in diets of animals and humans. Several medicinal plants or plant-derived natural bioactive molecules 
had been used for millennia for prevention and cure of many diseases, which are widely explored recently as antimicrobial agents and potent drug substances (Patra, 2012; Gechev et al., 2014; Atanasov et al., 2015). There are more than 350,000 PSC identified in several broad classified groups including alkaloids, glucosinolates, saponins, terpenes, flavonoids and phenylpropanoids (Wink, 2003; Patra and Saxena, 2010; Patra, 2012). Only few of them have been evaluated for their antimicrobial actions, stimulating effects on the digestive enzyme secretion, modulatory effects on ruminal microbial fermentation, antioxidant properties and various pharmacological effects in livestock animals (Windisch et al., 2008; Patra and Saxena, 2010, 2011; Patra and Yu, 2015; Sar et al., 2015; Kumar et al., 2017.)

Dietary PSC can also modulate the gastrointestinal (GI) integrity and health, and consequently modulate the nutrient transport and barrier functions in the GI tract (Patra et al., 2018a). The GI tract is inhabited by several trillion of commensal microbes in animals and humans, and they take part in the molecular crosstalk with the GI epithelial signaling pathways influencing GI health and barrier function (Wells et al., 2010; Ulluwishewa et al., 2011). Although the PSC are specifically applied to inhibit the growth of pathogenic microbiota in the GI tract, they may impart in many other beneficial biological roles. In fact, many PSC have shown promising in livestock animals to improve growth performance, to inhibit growth of pathogenic microorganisms in the intestine, to boost immunity, and to mitigate methane production and to improve quality of livestock-derived food products, which have been reviewed elsewhere (Windisch et al., 2008; Patra, 2014; Patra and Saxena, 2010; Kumar and Patra, 2017; Alagawany et al., 2018). Dietary PSC may potentially regulate nutrient transport mechanisms in the GI tract. The present review delineates the effects of different PSC on nutrient transport and their molecular mechanisms in the GI tract of farm animals.

\section{Physiology of nutrient transport systems in gut}

The mucosal surface of the GI tract is lined by a single layer of tightly connected columnar epithelial cells that segregate lamina propria from the intestinal lumen (Ulluwishewa et al., 2011). The intestinal epithelial cells are primarily composed of over $80 \%$ absorptive enterocytes along with goblet, Paneth and enteroendocrine cells (Ulluwishewa et al., 2011; Peterson and Artis, 2014). The mucosa performs nutrient absorption and waste secretion, and forms a highly dynamic barrier between the adverse external milieu of the lumen and the internal submucosal tissue in the intestine (Turner, 2009; Ulluwishewa et al., 2011). This function requires a selectively permeable barrier permitting the passage of nutrients, ions, minerals, water and preferred antigens, but restricting the entry of pathogens (bacteria, viruses and antigenic substances) and toxins, consequently modulating the epithelial nutrient transport and barrier function in the intestine (Ulluwishewa et al., 2011). 
Epithelial cells of the GI tract are tightly fastened at paracellular interfaces by intercellular junctional complexes, which are essential for regulation of paracellular permeability and preservation of barrier function (Turner, 2009; Ulluwishewa et al., 2011; Suzuki and Hara, 2011; Kosiñska and Andlauer, 2013). The intercellular junctional complexes, which are comprised of different protein motifs including tight junction (TJ), adherens junctions, gap junctions and desmosomes, maintain paracellular permeability interacting with cellular cytoskeleton (Turner, 2009; Ulluwishewa et al., 2011). The molecular structures of the junctional assembly are greatly dynamic, which are continually modified by various regulatory proteins depending upon the interactions with outside luminal stimuli including pathogenic and commensal microbiota, toxins and food residues (Ulluwishewa et al., 2011).

There are two pathways by which nutrients and other solutes can pass through the epithelial membrane: (1) the paracellular pathway, where passive diffusion of solutes occurs through water-filled pores of the TJ between the cells, and (2) the transcellular pathway, where nutrients essentially penetrate the enterocyte cell membranes. The TJ regulate passive movement of hydrophilic solutes including microorganisms, toxins and antigenic substances across the GI epithelium through the paracellular pathway (Ulluwishewa $e t$ al., 2011). Paracellular permeability has to be specifically regulated allowing nutrient movements while excluding the entry of pathogenic microorganisms and antigenic substances through the tight junctions. The absorption of nutrients through paracellular route is considered to be passive for hydrophilic solutes that cannot be transported via transcellular pathways, and thus requires increased TJ permeability, a high concentration of intralumenal nutrients, and a sufficient osmotic gradient to drive flow of fluid. However, the surface area for paracellular pores is very less constituting of $0.01 \%-0.1 \%$ of the total intestinal membrane surface area (Nellans, 1991). The transcellular pathway allows movements of molecules both passively and actively across the epithelial cell membranes, usually using specific transport or channel proteins (Turner, 2009). The transcellular transport is needed for efficient absorption and secretion of nutrients and ions and additionally creates a transepithelial concentration gradient to induce paracellular transport of nutrients, electrolytes and water passively (Clayburgh et al., 2006; Turner, 2009). Transcellular active nutrient absorption is mediated by transporter proteins expressed on enterocytes.

There are many transporter proteins for different nutrient absorption in different animals. For example, glucose absorption across the apical surface of the enterocytes is mediated primarily by the $\mathrm{Na}^{+}$-dependent glucose (also transports galactose) transporter 1 (SGLT1; a major intestinal glucose transporter) that is encoded by SLC5Al gene, whereas glucose and fructose 
transport across the basolateral side of enterocytes and into the blood circulation is mainly mediated by the facilitated transporter $\mathrm{Na}^{+}$-independent glucose transporter 2 (GLUT2), which is encoded by SLC2A2 (Röder et al., 2014; Williams et al., 2017). GLUT2 transporter is also localized at apical side of enterocytes and can also transport fructose, galactose, mannose and glucosamine. Fructose can also be transported by GLUT5 at the apical side of the cells.

Amino acids are absorbed in their free forms by different specific amino acid transporters or as di- and tripeptides by the peptide transporter (e.g., PEPT1) in different animals and humans. Amino acid transporters usually have substrate specificities. For instance, absorption of basic amino acids is mediated by BAT and $\mathrm{Na}^{+}$-independent cationic and zwiterionic amino acid transporter $\left(\mathrm{b}^{\mathrm{o}+} \mathrm{AT}\right)$, $\mathrm{Na}^{+}$-independent cationic amino acid transporter 1 (CAT1) and CAT2, $\mathrm{Na}^{+}-$ independent cationic and $\mathrm{Na}^{+}$-dependent neutral amino acid transporter $1\left(\mathrm{y}^{+} \mathrm{LAT} 1\right)$ and $\mathrm{y}^{+}$LAT2 transporters, neutral amino acids by $\mathrm{B}^{\circ} \mathrm{AT}$ and LAT1, and anionic amino acids by excitatory amino acid transporter 3 (EAAT3) and there are some overlaps in substrate specificities in these transporters (Gilbert et al., 2008; Mastrototaro et al., 2016).

\section{Effect of PSC on nutrient transport proteins}

Various PSC interact with nutrient transporters that may be stimulated or inhibited resulting in modulations of absorption of nutrients and drugs across GI tracts. The influences of PSC on transcellular nutrient transport mechanisms in the GI tract in farm animals are scanty. In a study with nematode (Ascaris suum) infected pigs, cinnamaldehyde was added to a diet at $1 \mathrm{~g} / \mathrm{Kg}$ diet or orally dosed with $1 \mathrm{~g} / \mathrm{d}$ on 11 and 13 day post-infection (Williams et al., 2017). Cinnamaldehyde supplemented with the diet, but not oral dosing of cinnamaldehyde, increased mRNA expressions of SLC5AI and tended to increase the expression of $S L C 2 A 2$. A recent nutrient uptake measurement study in Ussing chambers using epithelia obtained from sheep supplemented with mentholbased PSC (at 80 and $160 \mathrm{mg} / \mathrm{d}$ ) showed that $\mathrm{Na}^{+}$-independent glucose and methionine uptakes were increased from the rumen, but not from the intestine of sheep receiving the menthol-based PSC; whereas menthol did not affect $\mathrm{Na}^{+}$-dependent uptakes of glucose and methionine from rumen and intestine (Patra et al., 2018b). Genistein (a isoflavone present in soyabean) added to milk replacer (fed to piglet at dose rate of $360 \mathrm{~mL} / \mathrm{Kg}$ body weight) at 1 or $14 \mathrm{mg} / \mathrm{L}$ did not influence glucose or glutamine absorption in jejunum and ileum of piglets, which was measured by the change in short-circuit current $\left(I_{s c}\right)$ induced by the addition of either $10 \mathrm{mM}$ of glucose or glutamine to the luminal buffer in Ussing chambers (Chen et al., 2005). Genistein, however, reduced enterocyte proliferation and migration in the small intestine (Chen et al., 2005). Zhang et al. (2013) conducted a study to investigate the fermented or 
non-fermented Ginkgo biloba leaves on SGLT1 expression in duodenum with or without exposure to lipopolysaccharide (a stimulator of the intestinal immune system and present in the membrane of all gramnegative bacteria) in broiler chickens. Ginko leaves are rich sources of bioactive flavonoids, polysaccharides and terpenoids. Expression of SGLT1 was not affected by fermented or unfermented G. biloba leaves in broiler chickens without lipopolysaccharide-challenge, but fermented leaves decreased the SGLT1 expressions in lipopolysaccharidechallenged chickens compared with the control or non-fermented leaves (Zhang et al., 2013). From the overall results of this study, it was suggested that dietary fermented Ginko leaves may be useful for chickens, especially in the presence of stress, which can be partially ascribed to the immunomodulatory effect of flavonoids and polysaccharides of fermented leaves on maintaining Th1/Th2 balance in response to extracellular pathogens (Zhang et al., 2013).

In contrast to the stimulating effects on nutrient transporters, some PSC may inhibit the active transport of nutrients. Polyphenols are known to interact directly with glucose transporters to regulate the rate of glucose absorption. With in vitro cell culture study, for example, an anthocyanin (a flavonoid compound)-rich berry-extract $(0.125 \%, w / v)$ reduced sodium-dependent as well as sodium-independent (facilitated uptake) glucose uptake by human intestinal Caco- 2 cells, which was accompanied by down-regulation of SGLT1 mRNA and GLUT2 mRNA expression (Alzaid et al., 2013). Capsaicin decreased $\mathrm{Na}^{+}$-dependent methionine uptake in rat intestinal epithelial cells (IEC-6) in vitro, which was reported due to downregulation of sodium-coupled neutral amino acid transporter 2 (SNAT-2) mRNA levels and its SNAT2 protein at the apical membrane (Talukdar et al., 2016). Tomato seed saponin, tomatoside A (10 iM for $3 \mathrm{~h}$ ) reduced glucose transport by $46 \%$ in human intestinal Caco- 2 cells, which was supported by decreased expression of GLUT2, but there was no change in the expression of SGLT1 (Li et al., 2018). In this study, lower glucose uptake by tomatoside $\mathrm{A}$ was ameliorated by a protein kinase $\mathrm{C}$ inhibitor suggesting suppressed GLUT2 expression via PKC signaling in Caco- 2 cells.

Green tea polyphenols competitively inhibited the SGLT1 transport protein with greater inhibitory effects of epicatechin gallate in small intestinal brush-border membrane vesicles of rabbits (Kobayashi et al., 2000). In an Ussing chamber study using jejunal and ileal epithelia of pigs, a non-flavonoid polyphenol trans-resveratrol $(0.3 \mathrm{mM})$ and $\varepsilon$-viniferin $(0.3 \mathrm{mM})$ reduced glucose uptake (Guschlbauer et al., 2013). In a further detailed study, apical addition of trans-resveratrol $(0.3 \mathrm{mM})$ decreased $\mathrm{Na}^{+}$-dependent glucose and alanine transport in the jejunum and ileum of pigs which was associated with phosphorylation of SGLT1 and kinases because resveratrol increased the phosphorylation of SGLT1, protein kinase A substrates and AMP- 
activated protein kinase (Klinger and Breves, 2018). SGLT1, PEPT1 and phosphorylated $\mathrm{Na}^{+} / \mathrm{H}^{+}$-exchanger 3 did not change due to resveratrol in this study. Authors suggested that greater cAMP levels are likely a part of the mechanisms affecting the nutrient transport. Quercetin-3-Oglucoside decreased Na-dependent glucose uptake in a dose dependent and competitive manner, but did not affect L-leucine uptake into porcine jejunal brush border vesicles, which suggested a specific inhibition of SGLT1 by the quercetin glucoside (Ader $e t$ al., 2001; Cermak et al., 2004). In addition, quercetin-3-O-glucoside also reduced the Na-independent glucose uptake. Among other flavonoids tested (quercetin-4-Oglucoside,-3-O-galactoside, -3-Oglucorhamnoside and aglycone quercetin, naringenin-7-O-glucoside, genistein-7-Oglucoside and cyanidin-3,5-O-diglucoside), only quercetin-4-O-glucoside inhibited Nadependent glucose uptake into vesicles in this study (Cermak et al., 2004). Thus, dietary quercetin monoglucosides are competitive inhibitor to intestinal nutrient transporters such as SGLT1, possibly interacting the glucose moiety with the sugar binding site of the transporter (Cermak et al., 2004; Guschlbauer et al., 2013). In ruminants, quercetin glucoside may be deglycosylated by ruminal microorganisms, which would then show less inhibitory effect on glucose uptake in rumen and intestine. Nevertheless, flavonoid aglycones may also showed inhibitory effect on GLUT2 transporter as shown in a Caco-2E cell culture study (Kwon et al., 2007). Dietary PSC may also interact with mineral absorption in the intestine. As for example, oral administration of quercetin to rats decreased iron absorption in the duodenal mucosa and also the mRNA expression levels of iron transporters such as divalent metal transporter 1 (DMT1) and ferroportin (Lesjak et al., 2018). When interpreting the negative effects of resveratrol and quercetin on specific nutrient transport functions, it needs to be considered that resveratrol otherwise improved overall gut functionality by upregulating tight junction gene expression, reducing heat shock protein gene expression and permeability in heat-stressed broiler chickens (Liu et al., 2016; Zhang et al., 2017). Thus, the selection of a PSC for practical use in feed and pharmaceutical industries should thus consider the whole portfolio of expectable effects (Patra et al., 2018a).

\section{Effect of PSC on permeability of nutrients}

As pointed out before, permeability of the paracellular pathways is highly regulated by the TJ, which comprises of many proteins including claudins, occludin, and cadherins (Turner, 2009). Tight junctions are also controlled by other peripheral proteins (i.e., zona occludens), actomyosin ring and regulatory protein kinases (Turner, 2009; Ulluwishewa et al., 2011). Many studies have shown that PSC affect claudins, occludin, zona occludens and various regulatory kinases (Patra et al., 2018a), which can modify TJ functions and in turn permeability of nutrient via paracellular route (Suzuki, 2013). The transepithelial 
electrical resistance (TEER) in Ussing chamber studies indicates mainly paracellular permeability to ions with higher TEER values being associated with decreased GI permeability (Wijtten et al., 2011). Feeding of broiler chickens with dietary thyme essential oil $(0.5 \mathrm{~g} / \mathrm{Kg}$ diet for 35 days) increased TEER values in the intestine (Placha et al., 2014). Placha et al. (2015) observed that lower concentrations of sage essential oil $(0.1$ and $0.25 \mathrm{~g} / \mathrm{Kg})$ increased TEER values while high concentration $(0.5$ and $1 \mathrm{~g} / \mathrm{Kg}$ diet $)$ had opposite effect, which suggests that an optimum dose is important to obtain targeted effects.

In passive transcellular transports, solutes penetrate the apical membrane, which is followed by diffusion through the cytoplasm of the cell interior and the solute exits through the basolateral membrane of enterocytes. There is very less study how PSC affect passive transcellular transports. In a study by Sun et al. (2015), an essential oil mixture $(60 \mathrm{mg} / \mathrm{Kg}$ diet for 24 days) containing thymol $(25 \%)$ and carvacrol $(25 \%)$ was fed and the authors investigated the transcellular permeability to propranolol in mucosal-to-serosal direction, while permeability to rhodamine 123 was assessed in serosal-to-mucosal direction. This mixture reduced the increase of passive transcellular permeability to propranolol (mucosal-toserosal) caused by $C$. perfringens infection. However, no effect on the transcellular flux of the P-glycoprotein substrate rhodamine 123 (serosal-to-mucosal) was noted (Sun et al., 2015).
Several studies have also investigated the effects of PSC when applied acutely to tissues or cells. In an Ussing chamber study, short circuit current $\left(I_{s c}\right)$ indicates an active ion transport and provides an evidence of electrolyte-dependent absorption of glucose and amino acid in the GI tract (Wijtten et $a l ., 2011)$. In the Ussing chamber study of Boudry and Perrier (2008), thymol or cinnamaldehyde added to the mucosal compartment increased $I_{s c}$ in the jejunal mucosa of piglets in a dose-dependent manner with doses ranging 10 to $100 \mu \mathrm{M}$ indicating increased ion transport due to these essential oil, but TEER values were not affected. It was also noted that thymol effect on $I_{s c}$ was inhibited in low $\mathrm{Cl}^{-}$buffer and $\mathrm{HCO}_{3}^{-}$-free buffer and abolished in low $\mathrm{Cl}^{-} / \mathrm{HCO}_{3}^{-}$-free buffer (Boudry and Perrier, 2008). Genistein did not influence carbachol or serotonin-induced $\mathrm{Cl}^{-}$ secretion in jejunum and ileum of piglets as measured by the change in $I_{s c}$ induced by the addition of either $0.1 \mathrm{mM}$ of carbachol or serotonin to the serosal buffer in Ussing chambers (Chen et al., 2005).

\section{Effect of PSC on ion channels}

A number of transient receptor potential (TRP) channels (e.g., TRPM6, TRPM7, TRPV3, TRPV4 and TRPA1) are expressed in ruminal and intestinal epithelia of cattle and sheep (Rosendahl et al., 2016; Schrapers et al., 2018). These TRP channels are involved in transport of mono and divalent cations across epithelia of rumen and intestine. For example, $\mathrm{NH}_{4}^{+}, \mathrm{Na}^{+}, \mathrm{K}^{+}$ and $\mathrm{Ca}^{2+}$ are transported across ruminal 
epithelia through cation-selective TRPV3 and TRPA1 channels (Rosendahl et al., 2016; Schrapers et al., 2018). Evidence also suggests that TRPV5 and TRPV6 are involved in intestinal transport of $\mathrm{Ca}^{2+}$ (Dimke et al., 2011) and TRPM6 and TRPM7 are involved in intestinal and ruminal transport of $\mathrm{Mg}^{2+}$ (Dimke et al., 2011; Martens et al., 2018).

There is growing evidence that PSC can modulate TRP channels. For instance, Rosendahl et al. (2016) investigated the effects of cinnamaldehyde, menthol, thymol, and capsaicin on ruminal TRP channels and uptake of $\mathrm{NH}_{4}^{+}$in the ruminal epithelium. In ovine epithelia, menthol added apically in Ussing chamber at 10 and $100 \mu \mathrm{M}$ reduced $\mathrm{Ca}^{2+}$ flux from serosal to mucosal side (efflux), but tended to increase $\mathrm{Ca}^{2+}$ flux from mucosal to serosal side (influx) with net absorption of $\mathrm{Ca}^{2+}$. Menthol at high concentration $(1000 \mu \mathrm{M})$ did not influence influx of $\mathrm{Ca}^{2+}$; but increased efflux with a net decline of $\mathrm{Ca}^{2+}$ absorption (Rosendahl et al., 2016). Thymol at low concentrations $(10$ and $100 \mu \mathrm{M})$ had no noticeable effects on influx, efflux or net flux of $\mathrm{Ca}^{2+}$, but at high concentration (1000 $\mu \mathrm{M})$ increased both influx and efflux with net decline of $\mathrm{Ca}^{2+}$ absorption. In this study, menthol $(200$ and $1000 \mu \mathrm{M})$ increased the uptake of $\mathrm{NH}_{4}^{+}$, but cinnamaldehyde (1000 $\mu \mathrm{M})$ and capsaicin $(100 \mu \mathrm{M})$ decreased $\mathrm{NH}_{4}^{+}$uptake; whereas thymol (200 and 1000 $\mu \mathrm{M})$ did not affect $\mathrm{NH}_{4}^{+}$uptake in bovine ruminal epithelia (Rosendahl et al., 2016). In a patch-clamp technique study using bovine TRPV3-overexpressed HEK-293 cells, apical application of menthol, thymol and carvacrol at $1000 \mu \mathrm{M}$ enhanced whole cell currents mediated by $\mathrm{Na}^{+}, \mathrm{NH}_{4}^{+}$and $\mathrm{K}^{+}$, with an increase in intracellular $\mathrm{Ca}^{2+}$ concentration in response to menthol (Schrapers et al., 2018). Together, these results suggest that some essential oils could modulate activity of TRP channels acting as either agonists or antagonists depending upon concentrations and type of PSC, which consequently may affect the fluxes of various mono- or -divalent cations across the intestinal and ruminal epithelium.

\section{Conclusions}

Numerous PSC have been investigated for their use as feed additives, but only few of them have been evaluated for nutrient transport functions in the GI tract of farm animals. Dietary PSC may inhibit pathogenic microorganisms in the intestine, and improve GI epithelial barrier, they could simultaneously regulate nutrient absorption from rumen and intestine both positively and negatively depending upon the type of PSC and their doses. Therefore, there are challenges to select an effective dose at which a specific PSC could inhibit pathogenic microbial growth considerably while preserving or improving GI functions including nutrient and ion transports. A precise mechanism and an exact target site of action of the PSC at the molecular levels need to be unraveled to better understand the transport functions in the rumen and intestine for their applications in livestock feed formulation. 


\section{REFERENCES}

Ader P, Block M, Pietzsch S and Wolffram S, 2001. Interaction of quercetin glucosides with the intestinal sodium/glucose cotransporter (SGLT-1). Cancer Lett, 162: $17-180$

Alagawany M, Abd El-Hack ME, Farag MR, Shaheen MM and Abdel-Latif MA et al., 2018. The usefulness of oregano and its derivatives in poultry nutrition. World's Poult Sci J, 74: 463-474

Alzaid F, Cheung HM, Preedy VR and Sharp PA, 2013. Regulation of glucose transporter expression in human intestinal Caco-2 cells following exposure to an anthocyanin-rich berry extract. PLoS One, 8: e78932

Atanasov AG, Waltenberger B, PferschyWenzig EM, Linder T and Wawrosch C et $a l ., 2015$. Discovery and resupply of pharmacologically active plant-derived natural products: A review. Biotechnol Adv, 33: 1582-1614

Boudry G and Perrier C, 2008. Thyme and cinnamon extracts induce anion secretion in piglet small intestine via cholinergic pathways. J Physiol Pharmacol, 59: 543552

Cermak R, Landgraf S and Wolffram S, 2004. Quercetin glucosides inhibit glucose uptake into brush-border-membrane vesicles of porcine jejunum. Br J Nutr, 91: 849-855

Chen AC, Berhow MA, Tappenden KA and Donovan SM, 2005. Genistein inhibits intestinal cell proliferation in piglets. Pediatr Res, 57: 192-200
Chowdhury S, Mandal GP, Patra AK, Samanta I and Kumar P et al., 2018. Different essential oils in diets of broiler chickens: 2. Gut microbes and morphology, immune response, and some blood profile and antioxidant enzymes. Anim Feed Sci Technol, 236: 39-47

Clayburgh DR, Musch MW, Leitges M, Fu YX and Turner JR, 2006. Coordinated epithelial NHE3 inhibition and barrier dysfunction are required for TNF-mediated diarrhea in vivo. J Clin Invest, 116: 26822694

Dimke H, Hoenderop JG and Bindels RJ, 2011. Molecular basis of epithelial $\mathrm{Ca}^{2+}$ and $\mathrm{Mg}^{2+}$ transport: insights from the TRP channel family. J Physiol, 589: 1535-1542

Gechev TS, Hille J, Woerdenbag HJ, Benina $\mathrm{M}$ and Mehterov N et al., 2014. Natural products from resurrection plants: potential for medical applications. Biotechnol Adv, 32: 1091-1101

Gilbert ER, Li H, Emmerson DA, Webb KE Jr and Wong EA, 2008. Dietary protein quality and feed restriction influence abundance of nutrient transporter mRNA in the small intestine of broiler chicks. $\mathbf{J}$ Nutr, 138: 262-271

Guschlbauer M, Klinger S, Burmester M, Horn $\mathrm{J}$ and Kulling SE et al., 2013. transResveratrol and $\varepsilon$-viniferin decrease glucose absorption in porcine jejunum and ileum in vitro. Comp Biochem Physiol A Mol Integr Physiol, 165: 313-318

Klinger S and Breves G, 2018. Resveratrol inhibits porcine intestinal glucose and alanine transport: Potential roles of $\mathrm{Na}^{+} /$ 
$\mathrm{K}^{+}$-ATPase activity, protein Kinase A, AMP-activated protein kinase and the association of selected nutrient transport proteins with detergent resistant membranes. Nutrients, 10: 302

Kobayashi Y, Suzuki M, Satsu H, Arai S and Hara $\mathrm{Y}$ et al., 2000. Green tea polyphenols inhibit the sodiumdependent glucose transporter of intestinal epithelial cells by a competitive mechanism. J Agric Food Chem, 48: 56185623

Kosiñska A and Andlauer W, 2013. Modulation of tight junction integrity by food components. Food Res Int, 54: 951-960

Kumar P and Patra AK, 2017. Beneficial uses of black cumin (Nigella sativa L.) seeds as a feed additive in poultry nutrition. World's Poult Sci J, 73: 872-885

Kumar P, Patra AK, Mandal GP, Samanta I and Pradhan S, 2017. Effect of black cumin seeds on growth performance, nutrient utilization, immunity, gut health and nitrogen excretion in broiler chickens. J Sci Food Agric, 97: 3742-3751

Kwon O, Eck P, Chen S, Corpe CP and Lee JH et al., 2007. Inhibition of the intestinal glucose transporter GLUT2 by flavonoids. FASEB J, 21: 366-377

Lesjak M, Balesaria S, Skinner V, Debnam ES and Srai SKS, 2018. Quercetin inhibits intestinal non-haem iron absorption by regulating iron metabolism genes in the tissues. Eur J Nutr, doi: 10.1007/s00394018-1680-7

Li B, Terazono Y, Hirasaki N, Tatemichi Y and Kinoshita $\mathrm{E}$ et al., 2018. Inhibition of glucose transport by tomatoside A, a tomato seed steroidal saponin, through the suppression of GLUT2 expression in Caco2 cells. J Agric Food Chem, 66: 1428-1434

Liu L, Fu C, Yan M, Xie H and Li S et al., 2016. Resveratrol modulates intestinal morphology and HSP70/90, NF- $\kappa \mathrm{B}$ and EGF expression in the jejunal mucosa of black-boned chickens on exposure to circular heat stress. Food Funct, 7: 13291338

Martens H, Leonhard-Marek S, Röntgen M and Stumpff F, 2018. Magnesium homeostasis in cattle: absorption and excretion. Nutr Res Rev, 31: 114-130

Mastrototaro L, Sponder G, Saremi B and Aschenbach JR, 2016. Gastrointestinal methionine shuttle: Priority handling of precious goods. IUBMB Life, 68: 924-934

Nellans HN, 1991. Paracellular intestinal transport: modulation of absorption, Adv Drug Deliv Rev, 7: 339-364

Pathak M, Mandal GP, Patra AK, Samanta I and Pradhan S et al., 2016. Effects of dietary supplementation of cinnamaldehyde and formic acid on growth performance, intestinal microbiota and immune response in broiler chickens. Anim Prod Sci, 57: 821-827

Patra AK and Saxena J, 2009a. Dietary phytochemicals as rumen modifiers: a review of the effects on microbial populations. Antonie van Leeuwenhoek, 96: 363-375 
Patra AK and Saxena J, 2009b. The effect and mode of action of saponins on the microbial populations and fermentation in the rumen and ruminant production. Nutr Res Rev, 22: 204-219.

Patra AK and Saxena J, 2010. A new perspective on the use of plant secondary metabolites to inhibit methanogenesis in the rumen. Phytochemistry, 71: 1198-1222

Patra AK and Saxena J, 2011. Exploitation of dietary tannins to improve rumen metabolism and ruminant nutrition. J Sci Food Agric, 91: 24-37

Patra AK and Yu Z, 2015. Essential oils affect populations of some rumen bacteria in vitro as revealed by microarray (RumenBactArray) analysis. Front Microbiol, 6: 297

Patra AK, 2012. An overview of antimicrobial properties of different classes of phytochemicals. In: Dietary Phytochemicals and Microbes (Patra AK, ed), Springer, The Netherlands, pp 1-32

Patra AK, 2014. Exploring the benefits of feeding tannin containing diets for enhancing the nutritional values of milk and meat of ruminants. Indian J Anim Hlth, 53: 63-76

Patra AK, Amasheh S and Aschenbach JR, 2018a. Modulation of gastrointestinal barrier and nutrient transport function in farm animals by natural plant bioactive compounds - a comprehensive review. Crit Rev in Food Sci Nutr, doi:10.1080/ 10408398.2018

Patra AK, Geiger S, Braun HS and Aschenbach JR, 2018b. Influence of dietary menthol- based bioactive lipid compounds on conductance and uptakes of glucose and methionine in the rumen and intestine of sheep. $23^{\text {rd }}$ Symposium of the Specialist Group Physiology and Biochemistry of the German Veterinary Society, Veterinärmedizinische Universität Wien, 21-23 February 2018, Vienna, Austria, V25 (abstract)

Peterson LW and Artis D, 2014. Intestinal epithelial cells: regulators of barrier function and immune homeostasis. Nat Rev Immunol, 14: 141-153

Placha I, Ryzner M, Cobanova K, Faixova Z and Faix S, 2015. Effects of dietary supplementation with sage (Salvia officinalis L.) essential oil on antioxidant status and duodenal wall integrity of laying strain growers. Pol J Vet Sci, 18: 741-749

Placha I, Takacova J, Ryzner M, Cobanova K and Laukova A et al., 2014. Effect of thyme essential oil and selenium on intestine integrity and antioxidant status of broilers. Br Poult Sci, 55: 105-114

Röder PV, Geillinger KE, Zietek TS, Thorens B and Koepsell H et al., 2014. The role of SGLT1 and GLUT2 in intestinal glucose transport and sensing. PLoS One, 9: e89977

Rosendahl J, Braun HS, Schrapers KT, Martens $\mathrm{H}$ and Stumpff F, 2016. Evidence for the functional involvement of members of the TRP channel family in the uptake of $\mathrm{Na}^{+}$ and $\mathrm{NH}_{4}{ }^{+}$by the ruminal epithelium. Pflugers Arch, 468: 1333-1352

Sar TK, Patra AK, Samanta I and Mandal TK, 2015. Protective effect of a postential 
polyherbal drug on mammary tissue damage caused by intramammary administration of antibiotic by producing antioxidant action. Indian J Anim Hlth, 54: $35-42$

Schrapers KT, Sponder G, Liebe F, Liebe H and Stumpff F, 2018. The bovine TRPV3 as a pathway for the uptake of $\mathrm{Na}^{+}, \mathrm{Ca}^{2+}$ and $\mathrm{NH}^{4+}$. PLoS One, 13: e0193519

Sun Q, Liu D, Guo S, Chen Y and Guo Y, 2015. Effects of dietary essential oil and enzyme supplementation on growth performance and gut health of broilers challenged by Clostridium perfringens. Anim Feed Sci Technol, 207: 234-244

Suzuki T, 2013. Regulation of intestinal epithelial permeability by tight junctions. Cell Mol Life Sci, 70: 631-659

Suzuki, T, Tanabe S and Hara H, 2011. Kaempferol enhances intestinal barrier function through the cytoskeletal association and expression of tight junction proteins in Caco-2 cells. J Nutr, 141: 87-94

Talukdar J, Hill CC, Jaima A and Santiz-Lopez J, 2016. Capsaicin inhibits methionine cotransport in rat intestinal epithelial cells. FASEB J, 30 (suppl 1), abstract 420.3

Turner JR, 2009. Intestinal mucosal barrier function in health and disease. Nat Rev Immunol, 9: 799-809

Ulluwishewa D, Anderson RC, McNabb WC, Moughan PJ and Wells JM et al., 2011. Regulation of tight junction permeability by intestinal bacteria and dietary components. J Nutr, 141: 769-776
Wells JM, Rossi O, Meijerink $M$ and van Baarlen P, 2010. Epithelial crosstalk at the microbiota-mucosal interface. Proc Natl Acad Sci USA, 108 (Suppl 1): 4607-4614

Wijtten PJ, van der Meulen $\mathrm{J}$ and Verstegen MW, 2011. Intestinal barrier function and absorption in pigs after weaning: a review. Br J Nutr, 105: 967-981

Williams AR, Hansen TVA, Krych L, Ahmad HFB and Nielsen DS et al., 2017. Dietary cinnamaldehyde enhances acquisition of specific antibodies following helminth infection in pigs. Vet Immunol Immunopathol, 189: 43-52

Windisch W, Schedle K, Plitzner C and Kroismayr A, 2008. Use of phytogenic products as feed additives for swine and poultry. J Anim Sci, 86 (suppl): E140-E148

Wink M, 2003. Evolution of secondary metabolites from an ecological and molecular phylogenetic perspective. Phytochemistry, 64: 3-19

Zhang C, Zhao XH, Yang L, Chen XY and Jiang RS et al., 2017. Resveratrol alleviates heat stress-induced impairment of intestinal morphology, microflora, and barrier integrity in broilers. Poult Sci, 96: 43254332

Zhang X, Zhao L, Cao F, Ahmad H and Wang $\mathrm{G}$ et al., 2013. Effects of feeding fermented Ginkgo biloba leaves on small intestinal morphology, absorption and immunomodulation of early lipopolysaccharide-challenged chicks. Poult Sci, 92: 119-130 